\title{
Influential factors of 2-chlorobiphenyl reductive dechlorination by highly dispersed bimetallic nanoparticles
}

\author{
Junrong Jiang ${ }^{1,2}$, Chong $\mathrm{Fu}^{2}$ and Deming Zhao ${ }^{2 a}$ \\ ${ }^{1}$ School of Biological \& Chemical Engineering, Taizhou Vocational \& Technical College, Taizhou 318000, China \\ ${ }^{2}$ College of Chemical Engineering, Zhejiang University of Technology Hangzhou 310014, China
}

\begin{abstract}
Highly dispersed $\mathrm{Pd}-\mathrm{Fe}^{0}$ bimetallic nanoparticles were prepared in the presence of $40 \mathrm{kHz}$ ultrasonic irradiation in order to enhance disparity and reactivity, and simultaneously avoid agglomeration. Influential factors of 2-chlorobiphenyl (2-Cl BP) reductive dechlorination by highly dispersed $\mathrm{Pd}-\mathrm{Fe}^{0}$ nanoparticles were investigated. Experimental results showed that highly dispersed $\mathrm{Pd}_{-} \mathrm{Fe}^{0}$ nanoparticles prepared in the in the presence of ultrasound could further improve the dechlorination efficiency of 2-Cl BP, meanwhile the biphenyl (BP) formation rates increased obviously and increased from $47.4 \%$ (in the absence of ultrasound) to $95.3 \%$ (in the presence of ultrasound) within $300 \mathrm{~min}$. The catalytic reductive dechlorination effciency of 2-Cl BP was dependent on $\mathrm{Pd}_{-} \mathrm{Fe}^{0}$ nanoparticles prepared methods, $\mathrm{Pd}-\mathrm{Fe}^{0}$ nanoparticles dosage, $\mathrm{Pd}$ loading percentage over $\mathrm{Fe}^{0}$ and initial $\mathrm{pH}$ values
\end{abstract}

\section{Introduction}

Polychlorinated biphenyls (PCBs) are a sort of organic compounds of which there are 209 distinct chemical species known as congeners. PCBs as a class share a variety of properties (e.g., chemical and thermal stability) that made them popular for industrial applications in the latter part of the 20th century [1]. The high stability, low aqueous solubility, and high organic affinity of PCBs make them difficult to treat $[2,3]$. This raises an urgent need for efficient reductive dechlorination methods to eliminate chloroaromatics from both concentrated industrial effluents and diluted polluted groundwater.

Many remediation technologies,such as physical, chemical, and biological methods have been proposed, including dredging, landfilling, incineration, biodecomposition, in situ capping, and electrochemical degradation [2-4]. Among these technologies, the use of reactive metals such as nanoscale zero-valent iron (nZVI), as a mild and cost-effective reducing agent, has been documented to work efficiently for the reductive chlorinated organic compounds [5]. A rapid and complete catalytic reductive dechlorination method of chloroaromatics involving the use of bimetallic $\mathrm{Pd}-\mathrm{Fe}^{0}$ nanoparticles that led to the formation of non-chlorinated hydrocarbons have also been reported [6]. As one of the noble metal, $\mathrm{Pd}$ can utilize the produced $\mathrm{H}_{2}$ from $\mathrm{Fe}^{0}$ corrosion and accelerate the rates of dechlorination reaction [7].However, the presence of $\mathrm{Pd}$ not only reduces the accumulation of toxic byproducts, but also inhibits particle oxidation in air. $\mathrm{Pd}^{-} \mathrm{Fe}^{0}$ bimetallic nanoparticles, when compared to the conventional large particles have some advantages with possessing highly dispersion, large specific surface area and high surface reactivity [6]. In order to obtain the highly dispersed, stabilized and high reactive $\mathrm{Pd}^{-\mathrm{Fe}^{0}}$ bimetallic

\footnotetext{
a Corresponding author: dmzhao@zjut.edu.cn
}

nanoparticles and decrease their agglomeration and accumulation in the effluent, ultrasound is applied to the preparation of $\mathrm{Pd}-\mathrm{Fe}^{0}$ bimetallic nanoparticles. Sonochemistry arises from acoustic cavitation, the formation, growth, and implosive collapse of bubbles in a liquid. Acoustic cavitation can increase the surface area of the reactive solids by causing particles to rupture [8].

In the present work, Highly dispersed $\mathrm{Pd}-\mathrm{Fe}^{0}$ bimetallic nanoparticles were prepared in the presence of $40 \mathrm{kHz}$ ultrasound and influential factors of 2chlorobiphenyl (2-Cl $\mathrm{BP})$ reductive dechlorination by highly dispersed $\mathrm{Pd}-\mathrm{Fe}^{0}$ nanoparticles, such as $\mathrm{Pd}-\mathrm{Fe}^{0}$ nanoparticles availability, Pd content over $\mathrm{Fe}^{0}$, initial 2$\mathrm{Cl} \mathrm{BP}$ concentration, reaction temperature and the initial $\mathrm{pH}$ values, were investigated.

\section{Experimental}

\subsection{Chemicals}

2-chlorobiphenyl (AP grade), biphenyl (CP grade) and potassium hexachloropalladate (AP grade) were purchased from the J\&K Chemical Reagent Co., Ltd., China, $\mathrm{FeSO}_{4} \cdot 7 \mathrm{H}_{2} \mathrm{O}$ (AR grade), sodium borohydride (AR grade) purchased from Tianjin Chemical Reagent Research Institute. 2-Cl $\mathrm{BP}$ is dissolved in a methanol/deoxygenated deionized water solution (50:50, $V / V)$ and stored at $4{ }^{\circ} \mathrm{C}$. $\mathrm{Pd}^{-} \mathrm{Fe}^{0}$ nanoparticles were synthesized immediately before use.

\subsection{Experimental procedures}

In a $500 \mathrm{~mL}$ three-necked flask, $\mathrm{Pd}-\mathrm{Fe}^{0}$ bimetallic nanoparticles were prepared in the presence of $40 \mathrm{kHz}$ and $150 \mathrm{~W}$ ultrasound under nitrogen gas. Nanoscale zero-valent iron (nZVI) particles were synthesized by 
drop wise addition of stoichiometric amounts of $\mathrm{NaBH}_{4}$ aqueous solution into a flask containing $\mathrm{FeSO}_{4} \cdot 7 \mathrm{H}_{2} \mathrm{O}$ aqueous solution simultaneously with mechanical stirring at $25^{\circ} \mathrm{C}$. nZVI particles were then rinsed several times with deoxygenated deionized water. nZVI particles were then rinsed several times with deoxygenated deionized water. Subsequently, $\mathrm{Pd}-\mathrm{Fe}^{0}$ nanoparticles were prepared by reacting with the wet nZVI particles in an aqueous solution of potassium hexachloropalladate under mechanical stirring. Batch experiments of 2-Cl BP catalytic reductive dechlorination were performed in the same flask into which nanoscale bimetallic $\mathrm{Pd}-\mathrm{Fe}^{0}$ particles were added. 2-Cl BP stock solutions and a certain amount of deoxygenated deionized water and methanol solution $(50: 50, V / V)$ were added into the flask containing fresh prepared nanoscale $\mathrm{Pd}-\mathrm{Fe}^{0}$ particles into $500 \mathrm{~mL}$ of total reaction volume. The reaction solution was stirred under nitrogen flow to simulate anaerobic environment at $25{ }^{\circ} \mathrm{C}$. Aliquots of samples were periodically collected with glass syringes and the reaction was quenched by passing through $0.22 \mu \mathrm{m}$ polyether sulphone (PES) membrane filters.

\subsection{Methods of analysis}

All fresh prepared synthesized bimetallic $\mathrm{Pd}-\mathrm{Fe}^{0}$ nanoparticles (with Pd content 0.3 wt. \%) were immersed in absolute ethyl alcohol and dispersed by an ultrasonator. Brunauer-Emmett-Teller (BET) specific surface area of

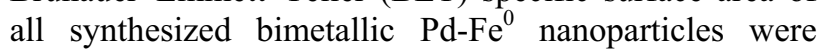
measured using nitrogen adsorption method with a surface analyzer (ASAR2020M+, Micromeritics Instrument Corp., US). Before the analysis, the particles were dried in vacuum at $25{ }^{\circ} \mathrm{C}$ for $24 \mathrm{~h}$ and then hydrogen flow at $260{ }^{\circ} \mathrm{C}$ for further $4 \mathrm{~h}$. Scanning electron microscope (SEM) images were obtained through a microscope (HITACHI S-4800 HITACHI Instruments Corp., JP). X-ray diffraction (XRD) analysis was performed by using $\mathrm{X}^{\prime}$ Pert Pro advanced X-ray diffractometer $\left(\lambda=1.5418 \mathrm{~A}^{\circ}\right)$. Organic compounds such as 2-chlorobiphenyl and BP were analyzed by Thermofish Trace 1310 Gas Chromatography. ECD and FID detector, Thermofish TR-5 Column $(30 \mathrm{~m} \times 0.32 \mathrm{~mm}$, $1.0 \mu \mathrm{m}$ ), inlet temperature $300{ }^{\circ} \mathrm{C}$, detector temperature $250{ }^{\circ} \mathrm{C}$, no split injection, injection volume $1 \mu \mathrm{L}$, carrier gas nitrogen (purity $\geq 99.99 \%$ ), column flow $1.5 \mathrm{~mL} / \mathrm{min}$ (constant current), program temperature: column temperature $100{ }^{\circ} \mathrm{C}$, holding $0.5 \mathrm{~min}, 25^{\circ} \mathrm{C} / \mathrm{min}$ was raised to $300{ }^{\circ} \mathrm{C}$, maintained $2 \mathrm{~min}$.

\section{Results and Discussions}

\subsection{Characterization of Pd-FeO Nanoparticles}

The BET specific surface area of $\mathrm{Pd}-\mathrm{Fe}^{0}$ nanoparticles prepared in the presence and absence of 40 $\mathrm{kHz}$ ultrasound were $41.68 \mathrm{~m}^{2} \mathrm{~g}^{-1}$ and $22.39 \mathrm{~m}^{2} \mathrm{~g}^{-1}$, respectively. The SEM images of the fresh synthesized $\mathrm{Pd}-\mathrm{Fe}^{0}$ nanoparticles in the presence of $40 \mathrm{kHz}$ ultrasound, and the aged (after $300 \mathrm{~min}$ ) $\mathrm{Pd}-\mathrm{Fe}^{0}$ nanoparticles prepared in presence of $40 \mathrm{kHz}$ ultrasound are shown in Fig. 1 (a, b). Fig. 1 (a) shows many Pd-Fe nanoparticles aggregate to form flocks and dendrites. After $300 \mathrm{~min}$ of reaction, the white platelet-shaped crystals appeared on the surface of $\mathrm{Pd}-\mathrm{Fe}^{0}$ nanoparticles and fraction of $\mathrm{Pd}-\mathrm{Fe}^{0}$ nanoparticle's diameter getting larger (Fig. 1(b)), suggests the formation of iron oxides resulting from iron corrosion. These minerals were likely composed of $\mathrm{FeOOH}$, or $\mathrm{Fe}_{3} \mathrm{O}_{4}$ [9]. This is generally agreement with our observation in XRD patterns. Fig. 1(e, f) shows the XRD patterns of the fresh and the $300 \mathrm{~min}$ reacted $\mathrm{Pd}-\mathrm{Fe}^{0}$ nanoparticles prepared in the presence of ultrasound. The XRD pattern for the fresh sample presents a strong peak $44.66^{\circ}$ which corresponds to the body-centered cubic $\mathrm{N}-\mathrm{Fe}^{0}$ at the (110) plane. The peak in the XRD pattern of the reacted sample shows evidence of iron oxides, possibly $\mathrm{Fe}_{3} \mathrm{O}_{4}$ or $\mathrm{Fe}_{2} \mathrm{O}_{3}$, or their mixture. This agrees with the previous work [10].

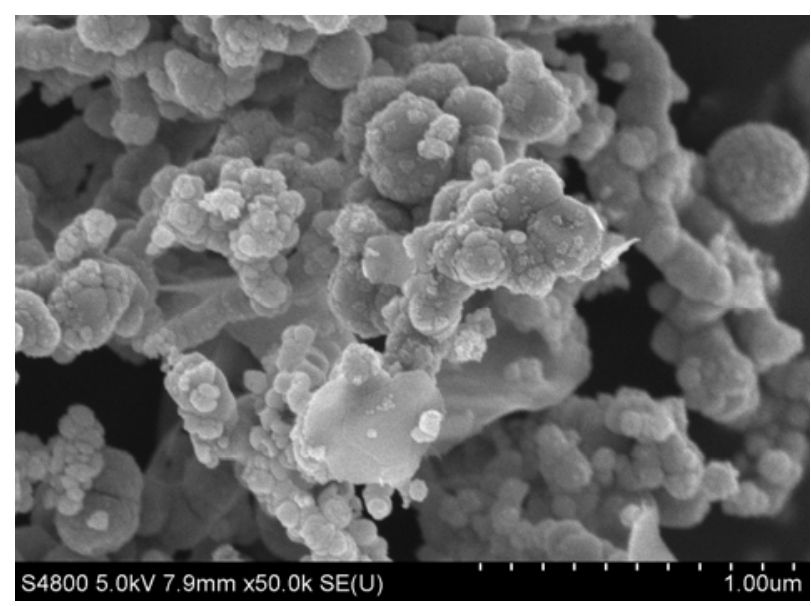

(a)

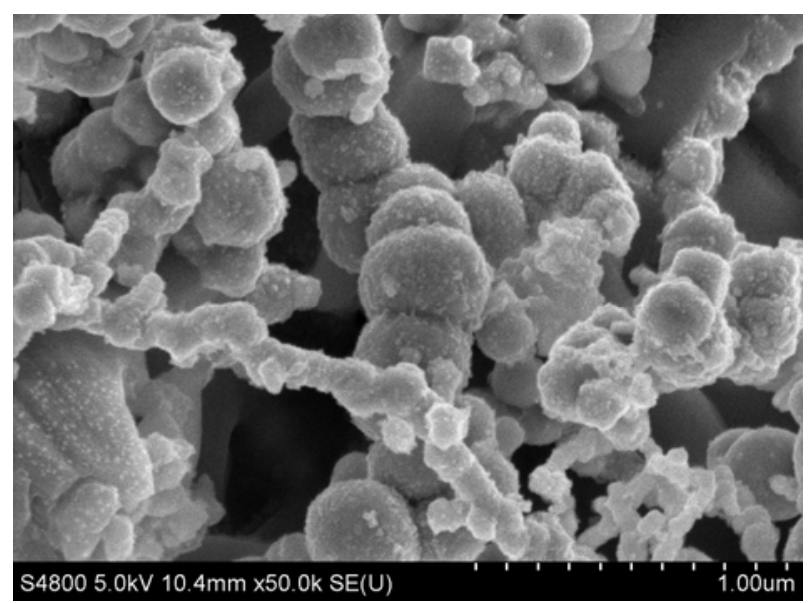

(b) 


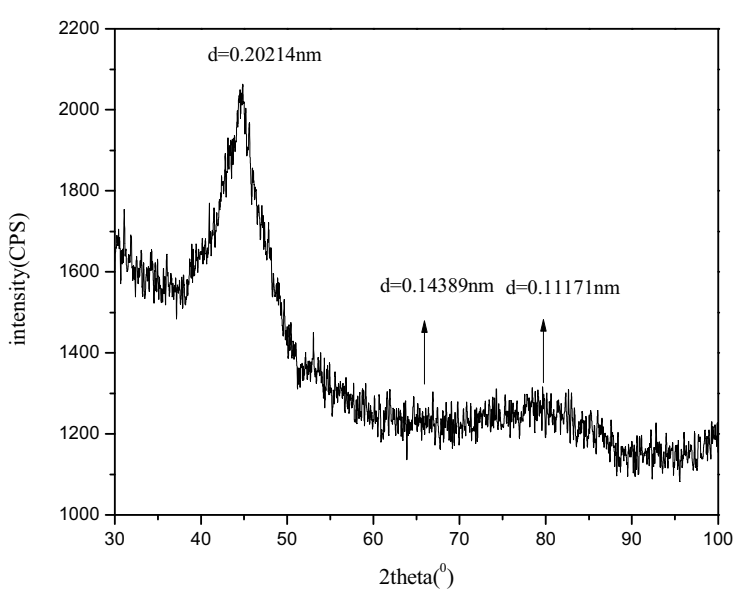

(c)

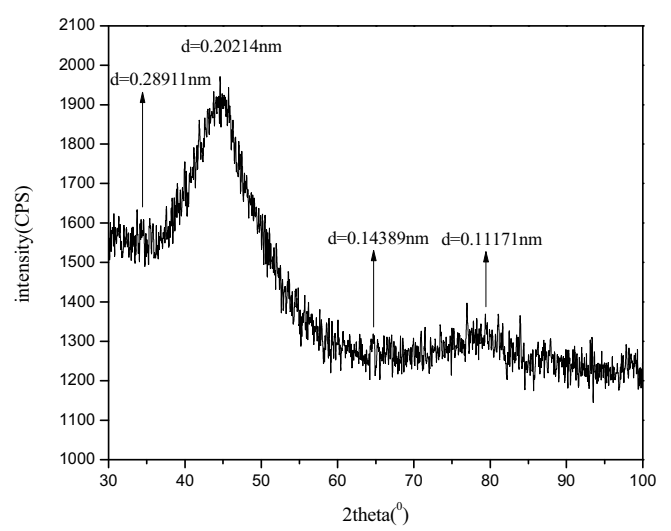

(d)

Figure 1. (a) SEM image of fresh synthesized Pd-Fe ${ }^{0}$ nanoparticles in the presence of $40 \mathrm{kHz}$ ultrasound, (b) SEM image of reacted $\mathrm{Pd}-\mathrm{Fe}^{0}$ nanoparticles synthesized in the presence of $40 \mathrm{kHz}$ ultrasound (300 min), (c) XRD patterns of fresh synthesized $\mathrm{Pd}-\mathrm{Fe}^{0}$ nanoparticles in the presence of $40 \mathrm{kHz}$ ultrasound and (d) XRD patterns of reacted $\mathrm{Pd}-\mathrm{Fe}^{0}$ nanoparticles synthesized in the presence of $40 \mathrm{kHz}$ ultrasound.

\subsection{Comparisons on 2-CI BP catalytic reductive dechlorinated efficiency by $\mathrm{Pd}-\mathrm{Fe}^{0}$ nanoparticles synthesized in different methods}

Effects of different $\mathrm{Pd}-\mathrm{Fe}^{0}$ nanoparticles prepared methods on 2-Cl BP catalytic reductive dechlorination were investigated at initial 2-Cl BP concentration of 10 $\mathrm{mg} \mathrm{L} \mathrm{L}^{-1},{\mathrm{Pd}-\mathrm{Fe}^{0}}^{0}$ nanoparticles dosage of $3 \mathrm{~g} \mathrm{~L}^{-1}, \mathrm{Pd}$ content of 0.8 wt. $\%$, initial $\mathrm{pH}$ value of 3.0 , reaction temperature of $25{ }^{\circ} \mathrm{C}$ and mechanical stirring speed of $600 \mathrm{rpm}$. Fig. 2 shows reductive dechlorinations of 2-Cl $\mathrm{BP}$ by $\mathrm{Pd}^{-\mathrm{Fe}^{0}}$ nanoparticles prepared in different methods .

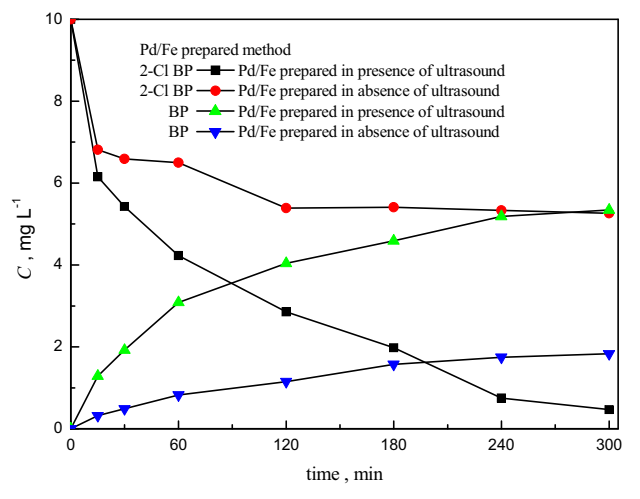

Figure 2. Effects of $\mathrm{Pd}-\mathrm{Fe}^{0}$ nanoparticles prepared methods on 2-Cl BP dechlorination

2-Cl BP was first adsorbed by $\mathrm{Pd}^{-} \mathrm{Fe}^{0}$ nanoparticles, then reduced to $B P$. No other chlorinated intermediates or final organic products were found. The concentration of 2-Cl $\mathrm{BP}$ decreased rapidly and the removal rate reached $80.2 \%$ in $180 \mathrm{~min}$, then further improved to nearly $95.3 \%$ in $300 \mathrm{~min}$ for $\mathrm{Pd}-\mathrm{Fe}^{0}$ nanoparticles prepared in the presence of $40 \mathrm{kHz}$ ultrasonic irridation. However, only about $45.8 \%$ and $47.4 \%$ of the removal rate were obtained for $\mathrm{Pd}-\mathrm{Fe}^{0}$ nanoparticles prepared in the absence of ultrasonic irridation at the same stages, respectively. Obviously, the catalytic reductive chloronation process quickly proceeded with bimetallic $\mathrm{Pd}-\mathrm{Fe}^{0}$ nanoparticles synthesized in the presence of ultrasonic irridation than with bimetallic $\mathrm{Pd}-\mathrm{Fe}^{0}$ nanoparticles prepared in the absence of ultrasonic irridation. So, highly dispersed bimetallic $\mathrm{Pd}-\mathrm{Fe}^{0}$ nanoparticles were synthesized in the presence of $40 \mathrm{kHz}$ ultrasonic irridation during the following experiments.

\subsection{Effects of $\mathrm{Pd}$ content over $\mathrm{FeO}$ on $2-\mathrm{Cl} \mathrm{BP}$ ruductive dechlorination}

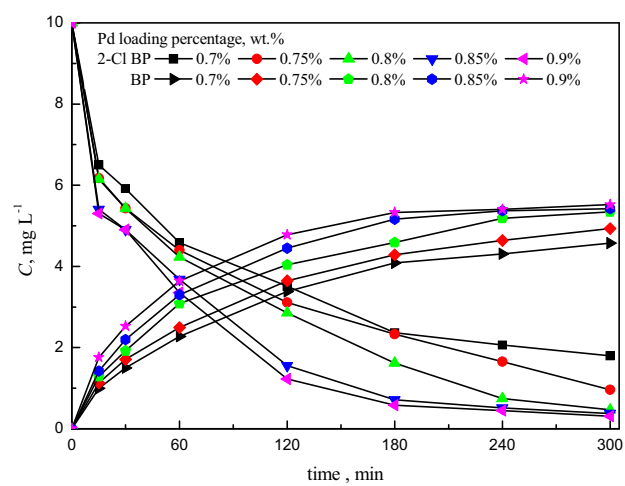

Figure 3. Effects of $\mathrm{Pd}$ loading content over $\mathrm{Fe}^{0}$ in $\mathrm{Pd}-$ $\mathrm{Fe}^{0}$ nanoparticles on 2-Cl BP dechlorination

$\mathrm{Fe}^{0}$ can enhance the hydrogenolysis reaction in which a chlorine atom in the organochlorides is replaced by a hydrogen atom. Palladium is an excellent catalyst for the hydrogenolysis reaction. Co-existence of $\mathrm{Pd}$ and $\mathrm{Fe}^{0}$ in the nanoparticles has been confirmed to be very 
effective to quicken the catalytic reductive dechlorination reaction. Then, the $\mathrm{Pd}$ content over $\mathrm{Fe}^{0}$ on $\mathrm{Pd}-\mathrm{Fe}^{0}$ nanoparticles may be one of the important influential factors on the reductive dechlorinated ratio. As Fig. 3 shows the efficiencies of reductive dechlorination and BP formations were obivously promoted as Pd content over $\mathrm{Fe}^{0}$ increased from 0.70 to $0.90 \mathrm{wt}$. \%. The removal ratio of 2-Cl BP reached from 82 to $97 \%$ within $300 \mathrm{~min}$. The increase in the Pd loading content over $\mathrm{Fe}^{0}$ from 0.75 to 0.85 wt. $\%$ only caused slight improvement to the $2-\mathrm{Cl}$ BP removal percentage. The most likely cause for this is that the maximum Pd coverage is less than one layer, and in this way, the loss of available catalytic reactive sites due to the overlapping between Pd atoms can be excluded. Nonetheless, the amount of hydrogen gas absorbed by Pd atoms promoted with the increasing Pd loaded content over $\mathrm{Fe}^{0}$ [11], and so make possible to accelerate the ratios of dechlorination and BP formations. The slight improvement of the removal ratio at Pd content over $\mathrm{Fe}^{0}>0.80$ wt. \% is that the accumulation of excessive hydrogen gas hinders the contact between 2-Cl BP and $\mathrm{Pd}-\mathrm{Fe}^{0}$ nanoparticles, and reduces the surface area available for 2-Cl BP dechlorination. This phenomenon is in agreement with previously reported studies $[6,10]$. Pd loading content over $\mathrm{Fe}^{0}$ in bimetallic $\mathrm{Pd}-\mathrm{Fe}^{0}$ nanoprticles was selected at about 0.80 wt. \% for optimized and efficient reductive dechlorination and yet minimal palladium content over $\mathrm{Fe}^{0}$.

\subsection{Effects of $\mathrm{Pd}-\mathrm{FeO}$ nanoparticles dose on 2-Cl BP reductive dechlorination}

Because the reductive dechlorination by $\mathrm{Pd}-\mathrm{Fe}^{0}$ nanoparticles happens on the surface of bimetallic $\mathrm{Pd}-\mathrm{Fe}^{0}$ nanoparticles, therefore bimetallic catalyst nanoparticles to 2-Cl BP ratio (g bimetallic Pd- $\mathrm{Fe}^{0}$ nanoparticles /mg 2$\mathrm{Cl} \mathrm{BP}$ ) is also an important influential factor. The quantity of available surface area is among the most significant experimental variables affecting 2-Cl BP reductive dechlorination efficiency. With increasing the dose of $\mathrm{Pd}-\mathrm{Fe}^{0}$ nanoparticles in the solution, it is insignificant to the final removal ratio in excess of $\mathrm{Pd}-\mathrm{Fe}^{0}$ nanoparticles dose. However, it will accelerate the initial reacted velocity and provide more active sites of $\mathrm{Pd}-\mathrm{Fe}^{0}$ nanoparticles for collision with 2-Cl BP during the reductive reaction. Effects of different $\mathrm{Pd}-\mathrm{Fe}^{0}$ nanoparticles dose on 2-Cl BP dechlorination were investigated as shown in Fig. 4. With the addition of the $\mathrm{Pd}-\mathrm{Fe}^{0}$ nanoparticles dose from 5 to $9 \mathrm{~g} \mathrm{~L}^{-1}$, obviously differences were detected, the removal ratio of 2-Cl BP increased from 63 to $91 \%$ after $180 \mathrm{~min}$ of the reaction. Increasing the $\mathrm{Pd} / \mathrm{Fe}$ nanoparticles dose means the larger calalysts surface area. The higher the catalysts surface area concentration is, the faster the reaction velocity. The removal ratio of 2-Cl BP is similar for a dose of $\mathrm{Pd}-\mathrm{Fe}^{0}$ nanoparticles of 7 or $8 \mathrm{~g} \mathrm{~L}^{-1}$ after $300 \mathrm{~min}$ of the reaction. Hence, the appropriate dose of $\mathrm{Pd}-\mathrm{Fe}^{0}$ nanoparticles in the level of $7 \mathrm{~g} \mathrm{~L}^{-1}$ is chosen for 2-Cl BP reductive dechlorination.

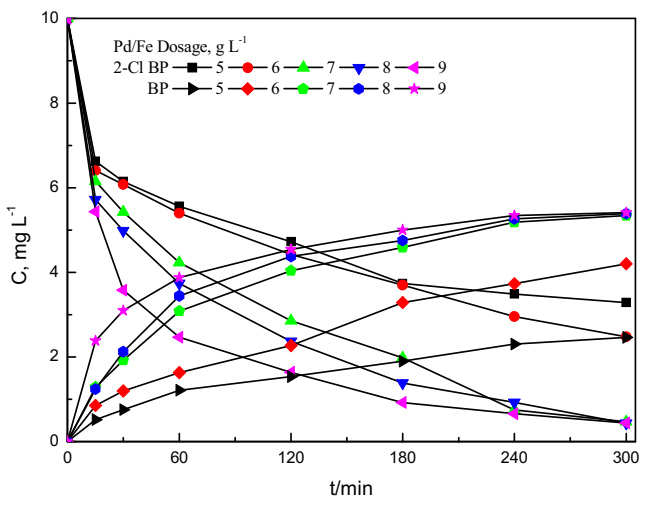

Figure 4. Effects of $\mathrm{Pd}-\mathrm{Fe}^{0}$ nanoparticles dose on 2-Cl BP dechlorination

\subsection{Effects of the initial $\mathrm{pH}$ value on $2-\mathrm{Cl} B P$ reductive dechlorination}

Initial $\mathrm{pH}$ value in aqueous solution is an important influential parameter in catalytic reductive dechlorination of organic chlorinated compounds using $\mathrm{Fe}^{0}$. Low $\mathrm{pH}$ prefers more $\mathrm{Fe}^{0}$ surface available for hydrogenolysis reaction with the chlorinated molecules or at least increase the corrosion velocity, leading to generate of chloride ions. Fig. 5 shows the effect of different initial $\mathrm{pH}$ values on the 2-Cl BP reductive dechlorination by bimetallic $\mathrm{Pd}-\mathrm{Fe}^{0}$ nanoparticles. Prior to initializing reaction, all reactant solutions were adjusted to different pH values by dilution with $\mathrm{H}_{2} \mathrm{SO}_{4}$ and $\mathrm{NaOH}$, and during the reaction $\mathrm{pH}$ values were not adjusted. When the initial $\mathrm{pH}$ values changed from 3, 5, 7 to 9 , the removal ratios of 2-Cl BP dropped from nearly 95.3, 70.6 to $54.8 \%$, respectively in $300 \mathrm{~min}$. The increase of initial $\mathrm{pH}$ values of the reactant solutions from 7 to 9 , leads to the reactant solution change from acidic to alkaline condition, consequently the removal ratios of 2-Cl BP dropped obviously. It shows that the presence of $\mathrm{H}^{+}$largely promotes the 2-Cl BP reductive dechlorination rate. The feasible reasons may be that (1) the surface of $\mathrm{Pd}-\mathrm{Fe}^{0}$ nanoparticles would be oxidized inevitably during the synthesis and storage, which had been confirmed by the earlier published literatures [12]. However, at lower $\mathrm{pH}$ values, the oxides on the catalyst particles surface were dissolved, and the active sites of the catalyst particle surface were exposed and it was benefical to the reductive reaction; (2) at lower $\mathrm{pH}$ values, $\mathrm{Fe}^{0}$ corrosion could be accelerated, producing enough hydrogen (or hydrogen atoms), which were benefical to hydrogenation reaction [13]; (3) Iron corrosion in solution of $\mathrm{pH}$ higher than 7 tends to form a passive film of iron oxides and hydroxide on $\mathrm{Fe}^{0}$ surface, which inhibits further reductive reaction. In the previous study $[10,14]$, detection of the solution $\mathrm{pH}$ during the entire period of the hydrogenation reaction, and ferrous ions/total iron ions produced in the reaction could further support our assumptions. 


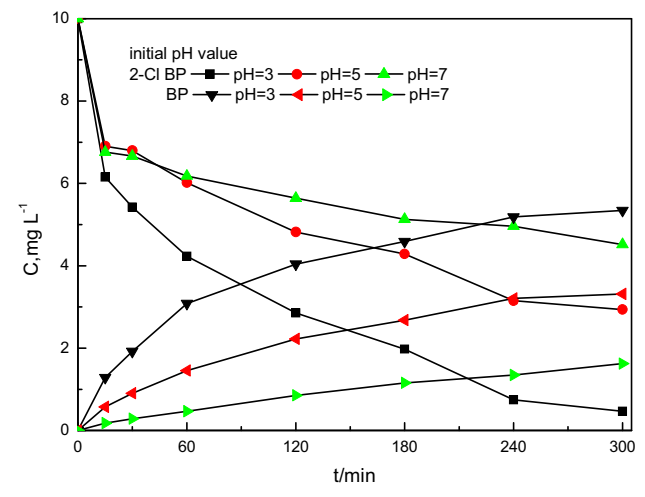

Figure 5. Effects of solution initial $\mathrm{pH}$ on 2-Cl BP dechlorination

\section{Conclusion}

The experimental results indicate that the prepared bimetallic $\mathrm{Pd}-\mathrm{Fe}^{0}$ nanoparticles in the presence of ultrasound was a better technique to enhance dispersion and the surface special properties. 2-Cl BP reductive dechlorination efficiency was dependent on a series of influential factors including highly dispersed bimetallic $\mathrm{Pd}-\mathrm{Fe}^{0}$ nanoparticles prepared method, $\mathrm{Pd}-\mathrm{Fe}^{0}$ nanoparticles dose, $\mathrm{Pd}$ loading content over $\mathrm{Fe}^{0}$ and initial $\mathrm{pH}$ values. $\mathrm{Up}$ to $95.0 \%$ of $2-\mathrm{Cl} \mathrm{BP}$ was removed after 300 min reaction with these experimental conditions: initial 2-Cl BP concentration $10 \mathrm{mg} \mathrm{L}^{-1}, \mathrm{Pd}$ loading content over $\mathrm{Fe}^{0} 0.8$ wt. \%, $\mathrm{Pd}-\mathrm{Fe}^{0}$ nanoparticles prepared in the presence of $40 \mathrm{kHz}$ ultrasonic irradiation available dose $7 \mathrm{~g} \mathrm{~L} \mathrm{~L}^{-1}$, initial $\mathrm{pH}$ value 3.0 , and reaction temperature $25{ }^{\circ} \mathrm{C}$. The removal ratio of $2-\mathrm{Cl} \mathrm{BP}$ degradation increased with the increasing $\mathrm{Pd}-\mathrm{Fe}^{0}$ nanoparticles dosage, and with the decrease of $\mathrm{pH}$ values.

\section{Acknowledgements}

The authors are grateful for the financial support provided by Zhejiang Provincial Natural Science Foundation of China (LY15B070005) and by the Science and Technology Department of Zhejiang Province (No. 2015C33233).

\section{References}

1. C.P. O'brien, M.C. Thies, D. A. Bruce, Environ. Sci. Technol. 39,6839-6844 (2005)

2. H. Choi, S. Agarwal, S.R. Al-abed, Environ. Sci. Technol. 43, 488-493 (2009)

3. J. Borja, D.M. Taleon, J. Auresenia, S. Gallardo, Process Biochem. 49, 1999-2013 (2005)

4. P. Murphy, A. Marquette, D.D. Reible, G.V. Lowry, J. Environ. Eng. 132, 787-794 (2006)

5. Y. Fang, S. R. Al-Abed, Environ. Sci. Technol. 41, 6253-6258 (2007)

6. Z. Zhang, Q.H. Shen, N. Cissoko, J.J. Wo, X.H. Xu, J. Hazard. Mater. 182, 252-258 (2010)
7. G.V. Lowry, M. Reinhard, Environ. Sci. Technol. 35, 696-702 (2001)

8. K.S. Suslick, M. Fang, T. Hyeon, J. Am. Chem. Soc., 118, 11960-11961 (1996)

9. D.H. Phillips, B. Gu, D.B. Watson, Y. Roh, L. Liang, S.Y. Lee, Environ. Sci. Technol.,34, 4169-4176 (2000)

10. D.M. Zhao, M. Li, D.X. Zhang, S.A.Baig, X.H. Xu, Ultrason Sono. 20, 864-871 (2013)

11. C. Grittini, M. Malcomson, Q. Farnando, N. Korte, Environ. Sci. Technol. 29, 2898-2900 (1995)

12. J.T. Nurmi, P.G. Tratnyek, V. Sarathy, D.R. Baer, J.E. Amonette, K. Pecher, C.M. Wang, J.C. Linehan, D.W. Matson, R.L. Penn, M.D. Driessen, Environ. Sci. Technol. 39, 1221-1230 (2005)

13. Y.H. Kim, E.R. Carraway, Environ. Sci. Technol. 34, 2014-2017 (2000)

14. D.M. Zhao, Y.Y. Zheng, M. Li, S.A.Baig, D.P.Wu, X.H. Xu, Ultrason Sono. 21, 1714-1721 (2014) 